\title{
Postoperative ascending aortic gigantic pseudoaneurysm: Endovascular treatment with the use of a septal occluder plug
}

\author{
MARCELLO ANDREA TIPALDI ${ }^{1}$, GIANLUIGI ORGERA ${ }^{1}$, MILTIADIS E. KROKIDIS $^{2, *}$, \\ FLORINDO LAURINO $^{1}$, FABIO CAPUANO ${ }^{3}$, MICHELE ROSSI $^{1}$ \\ ${ }^{1}$ Department of Radiology, Sant'Andrea University Hospital La Sapienza, Rome, Italy \\ ${ }^{2}$ Department of Radiology, Cambridge University Hospitals NHS Foundation Trust, Cambridge, UK \\ ${ }^{3}$ Department of Cardiac Surgery, Sant'Andrea University Hospital La Sapienza, Rome, Italy \\ ${ }^{*}$ Corresponding author: Miltiadis E. Krokidis, MD, PhD, EBIR, FCIRSE, FRCR; Consultant Vascular and \\ Interventional Radiologist, Department of Radiology, Cambridge University Hospitals NHS Trust, Hills Road, CB2 0QQ, \\ Cambridge, UK; Phone: +44 1223348 920; Fax: +44 1223217 847; E-mail: mkrokidis@hotmail.com
}

(Received: November 15, 2017; Revised manuscript received: July 8, 2018; Accepted: July 16, 2018)

\begin{abstract}
A rare postoperative complication of aortic root replacement is pseudoaneurysm formation. Surgical repair may be rather challenging particularly in patients who are elder and with significant comorbidities. Endovascular approach may also be technically demanding, given the high blood velocity and the anatomical challenges of the area of the aortic root and the ascending aorta. We would like to describe a case of an 85-year-old patient with history of prosthetic graft aortic root replacement who had been developed a 7 -cm pseudoaneurysm with sternotomy diastasis and extension in the subcutaneous tissue, 7 years after the initial operation. Given the comorbidities, open repair was not considered a valid option and successful endovascular repair with the use of a ventricular septal occluder plug followed. One-year follow-up confirmed satisfactory exclusion of the pseudoaneurysm with no migration of the endovascular device and no other complication. This is one of the rare cases on endovascular repair of an ascending aorta postoperative pseudoaneurysm.
\end{abstract}

Keywords: ascending aortic pseudoaneurysm, septal occluder plug, endovascular repair, aortic root replacement

\section{Introduction}

The pseudoaneurysm of the ascending aorta (PSAA) is high-risk complication that approximately occurs after $0.5 \%$ of cardiac surgery operations [1]. The likelihood of a pseudoaneurysm formation is higher when vascular grafts are used [1-2]. Surgical repair in such cases is associated with a very poor prognosis and high mortality that may reach up to $30 \%$ [3]. Endovascular approach is an established treatment method of pseudoaneurysms in other vascular territories; however, there is very limited experience in the ascending aorta, given the technical difficulties related to the elevated blood velocity and the heart movement.

We would like to describe a case of an 85-year old patient who developed a gigantic $(7 \mathrm{~cm})$ PSAA, 7 years after aortic root replacement, with sternotomy diastasis and extension in the subcutaneous tissue that was successfully treated endovascularly using ventricular septal occluder plug.

\section{Case Report}

An 85-year-old man was presented by his general practitioner complaining of the presence of an erythematous round lesion that was protruding from the surgical scar of his previous sternotomy. He mentioned that the lesion has been growing slowly over time and that lately was also associated with pain in the area. The patient has undergone aortic valve and aortic root replacement with the use of a prosthetic tube graft 7 years ago. The general

This is an open-access article distributed under the terms of the Creative Commons Attribution-NonCommercial 4.0 International License, which permits unrestricted use, distribution, and reproduction in any medium for non-commercial purposes, provided the original author and source are credited, a link to the CC License is provided, and changes - if any - are indicated. 
practitioner referred him to the cardiothoracic unit of our hospital; physical examination confirmed the presence of a non-pulsatile, erythematous round lesion in the cranial third of the previous sternotomy scar with a surrounding bruise (Fig. la and 1b).

Contrast-enhanced computed tomography (CECT) scan was performed and revealed a $7-\mathrm{cm}$ pseudoaneurysm arising from a $5-\mathrm{mm}$ fenestration of the distal suture line of the Dacron graft with significant amount of thrombus. The pseudoaneurysm was pushing the sutured sternum forward causing diastasis of the sternotomy and was extending anteriorly to the sternum in the subcutaneous tissue (Fig. Ic and Id).

The patient was discussed in the aortic multidisciplinary meeting and was considered as of high risk for surgical revision; an endovascular approach was suggested as an alternative option and was referred to interventional radiology.

The procedure was performed under local anesthesia. Written informed consent was obtained explaining the risks and benefits of the procedure, including the risk of potential pseudoaneurysm rupture during manipulation and potential embolic material migration in the cerebral circulation. A retrograde left femoral artery access was obtained and a $5 \mathrm{~F}$ sheath was inserted. Through a pigtail catheter, angiographic evaluation of the ascending aorta in anteroposterior and oblique views was followed in order to delineate the neck of the pseudoaneurysm. A second arterial access through the left axillary artery followed by another $5 \mathrm{~F}$ sheath was inserted. Using this access, the neck of the pseudoaneurysm was catheterized and a 0.035 in. standard hydrophilic guidewire was advanced into the pseudosac. The wire was then changed to a 0.035 in. Amplatz extra stiff guidewire (Cook Medical, Bloomington, IN, USA) and a 9F delivery sheath was introduced in the sac. An Amplatzer muscular ventricular septal defect occluder (St. Jude Medical, St. Paul, MN, USA) plug measuring $15 \times 7 \times 10 \mathrm{~mm}$ was advanced in the pseudoaneurysm neck and was successfully deployed. The final ascending aortogram confirmed the device position with the blockage of the to-and-fro flow into the pseudoaneurysm sac and with contrast stasis within the pseudoaneurysm cavity. The next day, the subcutaneous swelling was significantly reduced in size and a month later the skin wound was corrected surgically (Fig. 2a). CECT was further performed at $72 \mathrm{~h}$ after the procedure, at 1 and 6 months, and at 1 year after the procedure, and documented complete exclusion and reduction of the size of the pseudoaneurysm sac (Fig. 2b).

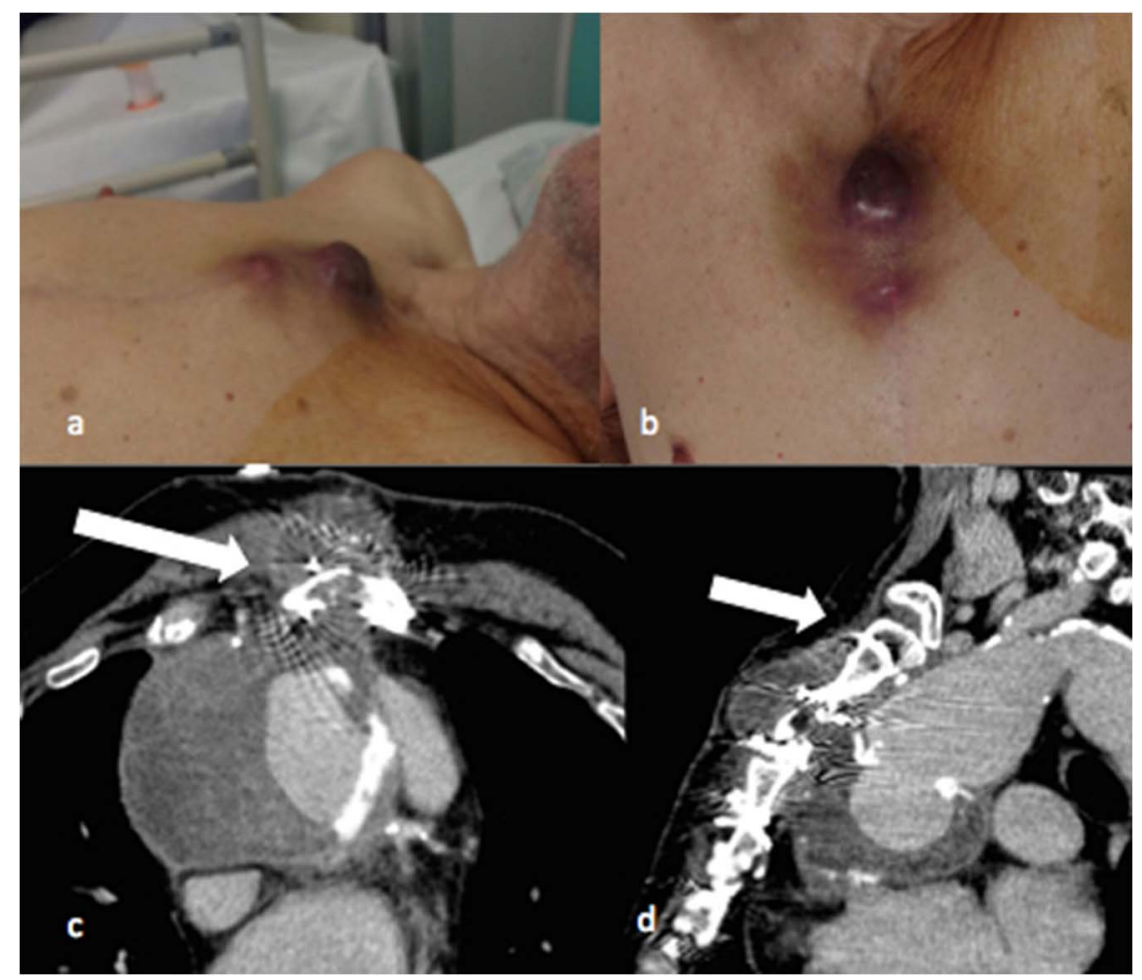

Fig. 1. Picture of an 85-year-old man, presenting with a non-pulsatile swelling cutaneous lesion in the upper third of the sternal wound. The lesion was surrounded by a bruise area ( $\mathrm{a}, \mathrm{b})$. CECT angiography scan revealed a large pseudoaneurysm measuring $70 \mathrm{~mm}$ in diameter arising from the distal suture line of the Dacron graft with a defect in the aortic wall of $5 \mathrm{~mm}$ and large parietal thrombus apposition (c, d). In addition, the sternum appeared with mild diastasis (arrow) and the pseudoaneurysm was abutting the sternum and extending through the bone to subcutaneous tissue. Anteriorly to the sternum was observed a fluid collection of $43 \mathrm{~mm}$ in connection with the pseudoaneurysm sac 


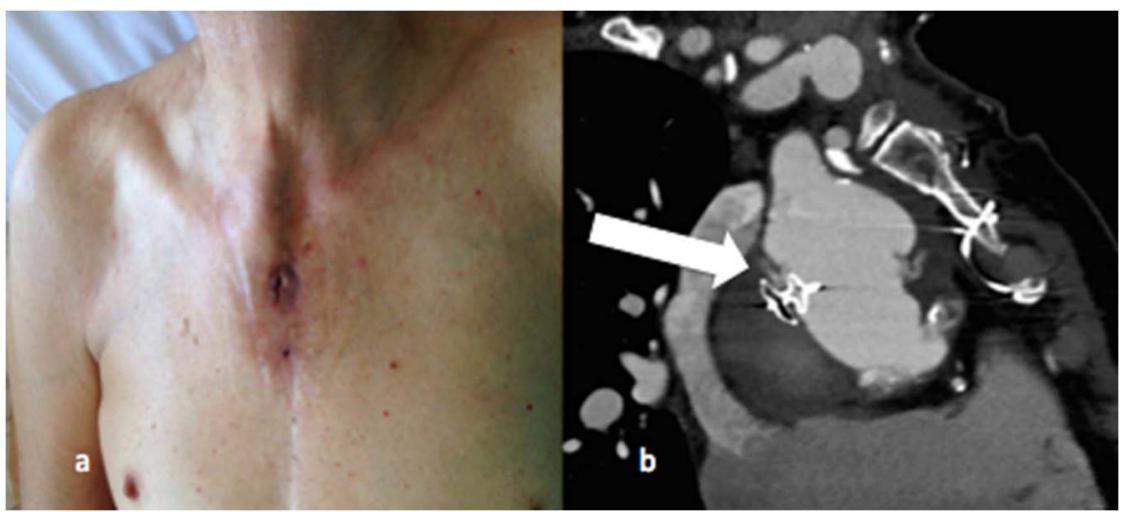

Fig. 2. (a) Macroscopic appearances of the lesion at 1 month after repair. (b) CECT performed at 1 -year follow-up documented totally exclusion of the sac. The vascular plug appears in position (arrow) and successful exclusion of the pseudoaneurysm from the flow is achieved

\section{Discussion}

The iatrogenic PSAA is a rare complication of major thoracic interventions; it is traditionally treated surgically with repeated ascending aorta ligation and graft replacement but with a high morbidity and mortality rate [3]. Endovascular techniques may offer a minimal invasive solution in other aortic territories using covered stents, liquid embolic, or coils [4]. However, there are some technical challenges in the ascending aorta, given the elevated blood velocity and the continuous heart movement that may lead to migration of the embolic material and lead to serious complication [5].

Very limited reports of successful endovascular repair of ascending aortic pseudoaneurysm appear in the literature. Bashir et al. [6] described the first case of an ascending aortic pseudoaneurysm treated percutaneously using an Amplatzer occluder device. Very few similar cases have been published since then as reported by a recent review from Patel et al. [7] reporting a median success rate of $80 \%$, over variable follow-up periods. The majority of these patients was of high risk, and was considered as not suitable for open surgery.

In the case described, the choice of the septal occluder device was the most appropriate in our view, given the anatomical complexity of the lesion. We retain that the use of other embolic material would have jeopardized the final result. In particular, the use of coils, even detachable ones, would have been linked to a migration risk through the pseudoaneurysm neck. Even if larger coils were used, a high number would have been required to exclude the whole pseudoaneurysm and would have been associated with a very high cost and an equivocal result, given the high flow of the area. Another potential option would have been the use of a proximal aortic cuff to cover the graft tear; however, given the short distance to the neck vessels and the lack of uniformity of the aortic root diameter, this was not considered as a valid option.
We would like to conclude that the use of an Amplatzer septal occluder has offered a valid minimal solution in a complex patient with a challenging problem, without complications and satisfactory during l-year follow-up.

Funding sources: None.

Authors' contribution: MAT and GO: data analysis and manuscript preparation. MEK: manuscript preparation and submission. FL, FC, and MR: manuscript editing.

Conflict of interest: The authors declare no conflict of interest.

\section{References}

1. Dumont E, Carrier M, Cartier R, Pellerin M, Poirier N, Bouchard D, Perrault LP: Repair of aortic false aneurysm using deep hypothermia and circulatory arrest. Ann Thorac Surg 78, 117-120 (2004)

2. Bical OM, Heran J, Thebault B, Funck F: Pseudoaneurysm following Dacron replacement of the ascending aorta. Eur J Cardiothorac Surg 35, 536 (2009)

3. D'Attellies N, Diemont FF, Julia PL, Cardon C, Fabiani JN: Management of pseudoaneurysm of the ascending aorta performed under circulatory arrest by port-access. Ann Thorc Surg 71, 1010-1011 (2001)

4. Fann JI, Samuels S, Slonim S, Burdon TA, Dalman RL: Treatment of abdominal aortic anastomotic pseudoaneurysm with percutaneous coil embolization. J Vasc Surg 35, 811-814 (2002)

5. Lin PH, Bush RL, Tong FC, Chaikof E, Martin LG, Lumsden AB: Intra-arterial thrombin injection of an ascending aortic pseudoaneurysm complicated by transient ischemic attack and rescued with systemic abciximab. J Vasc Surg 34, 939-942 (2001)

6. Bashir F, Quaife R, Carroll JD: Percutaneous closure of ascending aortic pseudoaneurysm using Amplatzer septal occluder device: The first clinical case report and literature review. Catheter Cardiovasc Interv 65, 547-551 (2005)

7. Patel AV, Gupta S, Laffin L, Retzer EM, Dill KE, Shah AP: One size does not fit all: Case report of two percutaneous closures of aortic pseudoaneurysm and review of the literature. Cardiovasc Revasc Med $15,160-164(2014)$ 